\title{
Regularization of star bodies by random hyperplane cut off
}

\author{
by \\ V. D. Milman (Ramat Aviv) and A. Pajor (Marne-la-Vallée) \\ To Professor Aleksander Pełczyński, to Olek, \\ with our great respect and admiration
}

\begin{abstract}
We present a general result on regularization of an arbitrary convex body (and more generally a star body), which gives and extends global forms of a number of well known local facts, like the low $M^{*}$-estimates, large Euclidean sections of finite volume-ratio spaces and others.
\end{abstract}

1. Introduction. In this paper, we consider random polytopes given by few linear inequalities. We first introduce a large class of probability measures, convenient for applications to random hyperplane cut off. For any $a \geq b>0$, let $M_{n}(a, b)$ be the set of symmetric probability measures $\mu$ on $\mathbb{R}^{n}$ satisfying, for all $t>0$ and $x \in \mathbb{R}^{n}$,

$$
\int e^{t(x, u)} d \mu(u) \leq e^{a^{2} t^{2}|x|^{2} / 2 n}, \quad \int|(x, u)|^{2} d \mu(u) \geq \frac{b^{2}|x|^{2}}{n} .
$$

The first inequality is of subgaussian type. It implies that for all $x \in \mathbb{R}^{n}$,

$$
\int|(x, u)|^{2} d \mu(u) \leq \frac{a^{2}|x|^{2}}{n} .
$$

Equivalently, for a random vector $X$ in $\mathbb{R}^{n}$, we write $X \in M_{n}(a, b)$ if the law of $X$ belongs to $M_{n}(a, b)$. Clearly, if $X \in M_{n}(a, b)$ then $X / b \in M_{n}(a / b, 1)$. It is sometimes convenient to introduce this parameter $b$, which is a matter of normalization. In applications, it will be mostly 1 , so that we will set $M_{n}(a)=M_{n}(a, 1)$. As a standard example of a measure in $M_{n}(1)$, we have in mind the Gaussian measure with covariance matrix $n^{-1}$ Id where Id denotes the identity matrix.

We use the following standard notation. The space $\mathbb{R}^{n}$ is equipped with the canonical Euclidean scalar product $(\cdot, \cdot)$ and the associated Euclidean

2000 Mathematics Subject Classification: 46B09, 52A20.

The first author was partially supported by the Israel Science Foundation founded by the Academy of Sciences and Hunamities. 
norm $|\cdot|$. Its unit ball is denoted by $D$. For any $1 \leq p<\infty$, the linear space $\mathbb{R}^{n}$ equipped with the norm $\left(\sum\left|x_{i}\right|^{p}\right)^{1 / p}$, for $x \in \mathbb{R}^{n}$, is denoted by $\ell_{p}^{n}$. Let $K$ be a convex body in $\mathbb{R}^{n}$ with 0 in its interior. Its polar $K^{\circ}$ is defined as usual by

$$
K^{\circ}=\left\{x \in \mathbb{R}^{n} ;(x, y) \leq 1 \text { for every } y \in K\right\} .
$$

The convex hull of a set $A \subset \mathbb{R}^{n}$ is denoted by $\operatorname{conv}(A)$. Although the article concerns mostly convex bodies, some results are valid for a larger class of star shaped bodies. A subset $A$ of $\mathbb{R}^{n}$ is star shaped or a star body if $\lambda x \in A$ for every $x \in A$ and $\lambda \in[0,1]$.

We define

$$
M_{K}^{*}=\int_{S^{n-1}} \sup _{x \in K}(x, t) d \sigma_{n}(t),
$$

where $\sigma_{n}$ denotes the rotation invariant probability measure on the sphere $S^{n-1}$. It is half of the mean width of $K$.

For every $u=\left(u_{1}, \ldots, u_{k}\right) \in\left(\mathbb{R}^{n}\right)^{k}$ and any $\alpha>0$, the set

$$
\mathrm{Cyl}_{k}(\alpha, u)=\bigcap_{1 \leq i \leq k}\left\{x \in \mathbb{R}^{n} ;\left(x, u_{i}\right) \leq \alpha\right\}
$$

will be called a $k$-cylinder. This is indeed a cylinder if $k \leq n$, the case we shall be interested in. If $X_{1}, \ldots, X_{k}$ are random vectors in $\mathbb{R}^{n}$, then

$$
\mathrm{Cyl}_{k}\left(\alpha, X_{1}, \ldots, X_{k}\right)
$$

will denote the random cylinder $\operatorname{Cyl}_{k}\left(\alpha, X_{1}(\cdot), \ldots, X_{k}(\cdot)\right)$.

Let $A$ and $B$ be two subsets of $\mathbb{R}^{n}$. The covering number $N(A, B)$ is defined as usual as

$$
N(A, B)=\min \left\{{ }^{\sharp} \Lambda ; \Lambda \subset \mathbb{R}^{n}, A \subset \Lambda+B\right\} .
$$

The volume of a measurable subset $A$ of $\mathbb{R}^{n}$ is denoted by $|A|$. Let $\sigma>0$ and let $K$ be a convex compact subset of $\mathbb{R}^{n}$ with 0 in its interior. We say that an ellipsoid $\mathcal{E}$ of $\mathbb{R}^{n}$ is an $M$-ellipsoid of $K$ with constant $\sigma$, or briefly an $M$-ellipsoid of $K$, if setting $\lambda=(|K| /|\mathcal{E}|)^{1 / n}$ in order that $|K|=|\lambda \mathcal{E}|$, we have

$$
N(K, \lambda \mathcal{E}) \leq e^{\sigma n}, \quad N\left(K^{\circ}, \lambda \mathcal{E}\right) \leq e^{\sigma n} .
$$

It is proved in [M1] (see also [M2] and [Pi] for simplified proofs) that there exists a universal constant such that for every $n$, every $n$-dimensional symmetric convex body has an $M$-ellipsoid with respect to this constant. An important feature of such ellipsoids is that they give reverse Brunn-Minkowski inequalities. Many interesting properties of centrally symmetric convex bodies and corresponding normed spaces have been revealed using $M$-ellipsoids. We refer to a survey [M3] and to an extension for non-centrally symmetric bodies in [M-P1]. 
Using a new approach and a simple deviation inequality of BernsteinChernov type, we prove the theorem below; the global form of so-called low $M^{*}$-estimates is one of its particular cases.

Theorem. Let $1 \leq k \leq n$. Let $a>0$ and let $X_{1}, \ldots, X_{k} \in M_{n}(a)$ be i.i.d. random vectors. There exist $\alpha=\alpha(a)>0$ and $\beta=\beta(a)>0$ such that for any compact star subset $K$ of $\mathbb{R}^{n}$ with 0 in its interior and so that $N(K, D) \leq e^{\alpha k}$, we have

$$
\operatorname{Cyl}_{k}\left(\frac{\beta}{4 \sqrt{k}}, X_{1}, \ldots, X_{k}\right) \cap K \subset \beta \sqrt{\frac{n}{k}} D
$$

with probability larger than $1-2 e^{-\alpha k}$.

This investigation was triggered by some question left open in [M-P3] concerning random regularization.

If a body $K$ satisfies $N(K, D) \leq e^{\alpha k}$ and $N\left(K^{\circ}, D\right) \leq e^{\alpha k}$ with $\alpha$ as above, then the Theorem applies to $K$ and its polar (Theorem 5). After $2 k$ operations, with $k$ small with respect to the dimension, first by taking the convex hull of $k$ points with Euclidean norm of order $\sqrt{k}$, and then by intersection with half-spaces of the type $(\cdot, u) \leq c / \sqrt{k}$, the body $K$ is transformed into a body which is close to the intersection of a Euclidean ball and a cylinder of the type $\operatorname{Cyl}_{k}\left(\beta / 4 \sqrt{k}, X_{1}, \ldots, X_{k}\right)$. There is of course a dual version, which transforms $K$ into a body which is close to the convex hull of a Euclidean ball and $k$ almost orthogonal vectors. We are thus faced with two new families of convex bodies (dual to each other), which seem to capture many of the properties of general convex bodies.

2. Preliminaries. The measures in $M_{n}(a, b)$ are almost isotropic (in the sense that the covariance matrix is not far from a multiple of the identity), normalized as the rotation invariant probability measure on the sphere (the variance of linear forms is of order $1 / n$ ), and linear forms associated to a unit vector have uniformly bounded Orlicz norm in the space $L_{\psi_{2}}(\mu)$, where $\psi_{2}(t)=\exp \left(t^{2}\right)-1, t \geq 0$.

Besides the Gaussian measure, the rotation invariant probability measure on the sphere belongs to $M_{n}(1)$. Another example is the uniform measure on the vertices of the cube $[-1 / \sqrt{n}, 1 / \sqrt{n}]^{n}$. Indeed, if $\mu$ is symmetric, then

$$
\int e^{t(x, u)} d \mu(u)=\int \cosh (t(x, u)) d \mu(u) \leq \int e^{t^{2}(x, u)^{2} / 2} d \mu(u) .
$$

Let $K$ be a centrally symmetric convex body in $\mathbb{R}^{n}$ and let $X$ be a uniformly distributed random vector on $K$. The body $K$ is called isotropic if it is of volume 1 and if the covariance matrix of $X$ (or the inertia tensor of $K$ ) is a multiple of the identity, so

$$
\mathbb{E}(x, X)^{2}=L_{K}^{2}|x|^{2}
$$


for any $x \in \mathbb{R}^{n}$, where $L_{K}$ is the so-called isotropic constant of $K$. It is unknown whether $(x, X)$ satisfies a $\psi_{2}$-estimate of type (1), with a uniform bound depending on dimension, even for a single $x$. But it is definitely not true for every $K$ and every $x$. It has been checked for some classes of convex bodies such as the isotropic ball of $\ell_{p}^{n}$ for $p \geq 2$ (see for example [B-K]) that a uniformly distributed random vector $X$ on $K$ belongs to $M_{n}\left(\alpha_{p} L_{K} \sqrt{n}, L_{K} \sqrt{n}\right)$ for some universal constant $\alpha_{p}>0$, and it is well known (see for example [M-P2]) that the isotropic constants are uniformly bounded over all $p \in[1, \infty]$. It is still an open problem whether the isotropic constants are uniformly bounded over the set of all convex bodies (see [M-P2] for a discussion and related problems).

However, in our main application, we do not need the strong assumption that any linear form satisfies a $\psi_{2}$-estimate (1). We use this only for some Euclidean $1 / 3$-net of $\ell_{2}^{n}$. We do not know how much this increases the family of convex bodies to which our results are applicable for uniform volume distribution on these bodies.

Bobkov and Nazarov [B-N1] showed that a $\psi_{2}$-estimate like (1) holds for a random vector uniformly distributed on an unconditional convex body (symmetric with respect to each coordinate hyperplane), for the direction $(1,1, \ldots, 1)$. A stronger and more precise result was proved recently by the same authors in [B-N2]. They show that, except for a set of "small" measure on the sphere, in the worst case, the tails of linear functionals are "almost" Gaussian.

Let $X_{i} \in M_{n}(a, b), i=1, \ldots, k$, be a collection of independent random vectors. Then it is clear from (1) that for any real numbers $\left(\alpha_{i}\right)$,

$$
\sum_{1 \leq i \leq k} \alpha_{i} X_{i} \in M_{n}(a \alpha, b \alpha) \quad \text { with } \quad \alpha^{2}=\sum_{1 \leq i \leq k} \alpha_{i}^{2} .
$$

Let $X \in M_{n}(a, b)$. Then it is easy to check (and classical) that for any $x \in S^{n-1}$,

$$
\mathbb{P}((x, X) \geq u) \leq e^{-n u^{2} / 2 a^{2}} \quad \text { for any } u \geq 0 .
$$

\section{Random hyperplane cut}

Lemma 1. Let $a, b>0$ and $X$ be a random vector in $M_{n}(a, b)$.

(i) For any non-zero vector $x \in \mathbb{R}^{n}$ and any $v \in(0,1)$, we have

$$
\mathbb{P}\left[(x, X)>v\left(\mathbb{E}(x, X)^{2}\right)^{1 / 2}\right] \geq c(a, b)\left(1-v^{2}\right)^{2}
$$

for some function $c(a, b)$ depending only on $a$ and $b$.

(ii) Let $1 \leq k \leq n$ be integers, $t>0$ and $X_{1}, \ldots, X_{k}$ independent copies of $X$. Then 


$$
\mathbb{P}\left[|x|^{2} \geq t^{2} \sum_{1 \leq i \leq k}\left|\left(x, X_{i}\right)\right|^{2} \text { for all } x \in \mathbb{R}^{n}\right] \geq 1-e^{-n / 18 a^{2} t^{2}+2(n+k)} .
$$

Proof. (i) Let $X \in M_{n}(a, b)$. By applying the decomposition

$$
\mathbb{E}\left((x, X)^{2}\right)=\mathbb{E}\left((x, X)^{2} \mathbb{1}_{(x, X)^{2}>s^{2}}\right)+\mathbb{E}\left((x, X)^{2} \mathbb{1}_{(x, X)^{2} \leq s^{2}}\right)
$$

with $s=v\left(\mathbb{E}(x, X)^{2}\right)^{1 / 2}$, we get $\left(1-v^{2}\right) \mathbb{E}\left((x, X)^{2}\right) \leq \mathbb{E}\left((x, X)^{2} \mathbb{1}_{(x, X)^{2}>s^{2}}\right)$. By the Cauchy-Schwarz inequality, we have

$$
\left(1-v^{2}\right) \mathbb{E}\left((x, X)^{2}\right) \leq\left(\mathbb{E}(x, X)^{4}\right)^{1 / 2}\left(\mathbb{P}\left[(x, X)^{2}>v^{2} \mathbb{E}\left((x, X)^{2}\right)\right]\right)^{1 / 2}
$$

(we could use a Hölder inequality and optimize to get better numerical constants). To estimate $\mathbb{E}(x, X)^{4}$, we use (1) with the choice $t=2 \sqrt{n} / a|x|$ to get $\mathbb{E}(x, X)^{4} \leq 12 a^{4}|x|^{4} / n^{2}$. Combining these estimates and using the symmetry of the random variable, we conclude that

$$
\mathbb{P}\left[(x, X)>v\left(\mathbb{E}(x, X)^{2}\right)^{1 / 2}\right] \geq \frac{\left(1-v^{2}\right)^{2}}{2} \cdot \frac{\left(\mathbb{E}(x, X)^{2}\right)^{2}}{\mathbb{E}\left((x, X)^{4}\right)} \geq \frac{b^{4}\left(1-v^{2}\right)^{2}}{24 a^{4}}
$$

for any $v \in(0,1)$.

(ii) Let $\left(e_{i}\right)$ be the canonical basis of $\ell_{2}^{k}$. Let $G: \ell_{2}^{k} \rightarrow \ell_{2}^{n}$ be the random operator defined by $G e_{i}=X_{i}$ for all $i=1, \ldots, k$. We note first that

$$
\Omega=\left\{\omega ;|x|^{2} \geq t^{2} \sum_{1 \leq i \leq k}\left|\left(x, X_{i}(\omega)\right)\right|^{2} \text { for all } x \in \mathbb{R}^{n}\right\}=\{\omega ;\|G(w)\| \leq 1 / t\} .
$$

Now let $\Lambda(k)$ and $\Lambda(n)$ be $1 / 3$-nets of the unit balls of $\ell_{2}^{k}$ and $\ell_{2}^{n}$ in their own metrics and with cardinality less than $7^{k}$ and $7^{n}$ respectively (see for example $[\mathrm{M}-\mathrm{S}])$. We have

$$
\|G\| \leq 3 \max \{(x, G y) ; x \in \Lambda(n), y \in \Lambda(k)\} .
$$

Property (2) gives

$$
G y=\sum_{1 \leq i \leq k} y_{i} X_{i} \in M_{n}(a, b)
$$

where $y=\sum_{1 \leq i \leq k} y_{i} e_{i} \in S^{k-1}$. By applying inequality (3) to $X=G y$ with $x \in S^{n-1}$, we conclude that

$$
1-\mathbb{P}(\Omega) \leq 7^{n+k} e^{-n / 18 a^{2} t^{2}} \leq e^{-n / 18 a^{2} t^{2}+2(n+k)} .
$$

REMARK. Suppose $k \leq n$. Then choosing $t=1 / a \sqrt{90}$ in the previous inequality yields $1-\mathbb{P}(\Omega) \leq e^{-n / 18 a^{2} t^{2}+2(n+k)} \leq e^{-n}$. Therefore

$$
\mathbb{P}\left[|x|^{2} \geq \frac{1}{90 a^{2}} \sum_{1 \leq i \leq k}\left|\left(x, X_{i}\right)\right|^{2} \text { for all } x \in \mathbb{R}^{n}\right] \geq 1-e^{-n} .
$$


4. Regularization of star bodies. Our main technical tool is:

Theorem 2. Let $0<k \leq n$ be integers and $a>0$. There exist $c(a)>0$ and $c^{\prime}(a)>0$, depending only on $a$, such that for every $x_{0} \in \mathbb{R}^{n}, x_{0} \neq 0$, every $X \in M_{n}(a)$ and any $k$ independent copies $X_{1}, \ldots, X_{k}$ of $X$, we have

$$
\operatorname{Cyl}_{k}\left(\frac{\left|x_{0}\right|}{4 \sqrt{n}}, X_{1}, \ldots, X_{k}\right) \cap B\left(x_{0}, c^{\prime}(a)\left|x_{0}\right| \sqrt{\frac{k}{n}}\right)=\emptyset
$$

with probability larger than $1-2 e^{-k c(a)}$.

Remark. Since $k \leq n, \mathrm{Cyl}_{k}\left(v\left|x_{0}\right| / 2 \sqrt{n}, X_{1}, \ldots, X_{k}\right)$ is a cylinder and when the measure has no atom, the vector space $\bigcap_{1 \leq i \leq k}\left\{x ;\left(x, X_{i}\right)=0\right\}$ is almost surely of codimension $k$.

Proof of Theorem 2. Let $x_{0} \in \mathbb{R}^{n}, x_{0} \neq 0$ and $X \in M_{n}(a)$, and let $X_{1}, \ldots, X_{k}$ be independent copies of $X$. Let $Z$ be a $0-1$ valued random variable so that

$$
p=\mathbb{P}[Z=1]=\mathbb{P}\left\{\left(x_{0}, X\right)>v\left|x_{0}\right| / \sqrt{n}\right\} .
$$

From Lemma 1 and (1), we see that for $0<v<1$,

$$
p \geq \mathbb{P}\left[\left(x_{0}, X\right)>v\left(\mathbb{E}\left(x_{0}, X\right)^{2}\right)^{1 / 2}\right] \geq c(a, 1)\left(1-v^{2}\right)^{2} .
$$

For any integer $m, 1 \leq m \leq k$, set

$$
\begin{aligned}
& \Omega_{m}\left(x_{0}, v\right) \\
& =\left\{\omega ;\left(x_{0}, X_{i}(\omega)\right)>v\left|x_{0}\right| / \sqrt{n} \text { for at least } m \text { indices } i \in\{1, \ldots, k\}\right\} .
\end{aligned}
$$

Hence, if $\omega \in \Omega_{m}\left(x_{0}, v\right)$, then there is a subset $I$ of $\{1, \ldots, k\}$ with cardinality $m$ such that

$$
\left(x_{0}, X_{i}(\omega)\right)>v\left|x_{0}\right| / \sqrt{n} \quad \text { for all } i \in I .
$$

Suppose moreover that $\omega$ is such that

$$
|z| \geq t\left(\sum_{i \in I}\left|\left(z, X_{i}(\omega)\right)\right|^{2}\right)^{1 / 2} \quad \text { for all } z \in \mathbb{R}^{n} .
$$

Let $x \in \mathrm{Cyl}_{k}\left(v\left|x_{0}\right| / 2 \sqrt{n}, X_{1}(\omega), \ldots, X_{k}(\omega)\right)$. This means

$$
\left(x, X_{i}(\omega)\right) \leq \frac{v\left|x_{0}\right|}{2 \sqrt{n}} \quad \text { for all } i=1, \ldots, k .
$$

Assuming the validity of (8)-(10) yields

$$
\left|\left(x-x_{0}, X_{i}(\omega)\right)\right| \geq\left(x_{0}, X_{i}(\omega)\right)-\left(x, X_{i}(\omega)\right) \geq \frac{1}{2} v\left|x_{0}\right| \frac{1}{\sqrt{n}}
$$

for every $i \in I$ and

$$
\left|x-x_{0}\right| \geq t\left(\sum_{i \in I}\left|\left(x-x_{0}, X_{i}(\omega)\right)\right|^{2}\right)^{1 / 2} \geq \frac{1}{2} t v\left|x_{0}\right| \sqrt{\frac{m}{n}} .
$$


Therefore

$$
\mathrm{Cyl}_{k}\left(\frac{v\left|x_{0}\right|}{2 \sqrt{n}}, X_{1}(\omega), \ldots, X_{k}(\omega)\right) \cap B\left(x_{0}, \frac{t v\left|x_{0}\right|}{2} \sqrt{\frac{m}{n}}\right)=\emptyset .
$$

It is left to estimate the probability that the relations (8) and (9) hold. We first use (5) to handle (9). Next we observe that if $Z_{1}, \ldots, Z_{k}$ are $k$ i.i.d. copies of $Z$, then

$$
1-\mathbb{P}\left(\Omega_{m}\left(x_{0}, v\right)\right)=\mathbb{P}\left[Z_{1}+\ldots+Z_{k}<m\right] .
$$

From the Chernov inequality, for $m \leq k p$, we have

$$
\mathbb{P}\left[Z_{1}+\ldots+Z_{k}<m\right] \leq e^{-2 k(p-m / k)^{2}} .
$$

We choose $m=k p / 2$ in the last estimate. This allows us to control the probability that (8) happens:

$$
1-\mathbb{P}\left(\Omega_{m}\left(x_{0}, v\right)\right) \leq e^{-k p^{2} / 2} .
$$

Now choose $v=1 / 2$; then $p \geq c(a, 1)\left(1-v^{2}\right)^{2} \geq(9 / 16) c(a, 1)$. This completes the proof as we can choose $t$ such that

$$
\operatorname{Cyl}_{k}\left(\frac{v\left|x_{0}\right|}{2 \sqrt{n}}, X_{1}(\omega), \ldots, X_{k}(\omega)\right) \cap B\left(x_{0}, \frac{t v\left|x_{0}\right|}{2} \sqrt{\frac{k p}{2 n}}\right)=\emptyset
$$

with probability larger than $1-e^{-k p^{2} / 2}-e^{-n / 18 a^{2} t^{2}+2(n+k)} \geq 1-2 e^{-k c(a)}$ for some function $c(a)$ depending only on $a$.

Theorem 3. Let $1 \leq k \leq n$. Let $a>0$ and let $X_{1}, \ldots, X_{k} \in M_{n}(a)$ be i.i.d. random vectors. There exist $\alpha=\alpha(a)>0$ and $\beta=\beta(a)>0$ such that for any compact star subset $K$ of $\mathbb{R}^{n}$ with 0 in its interior and so that $N(K, D) \leq e^{\alpha k}$, we have

$$
\operatorname{Cyl}_{k}\left(\frac{\beta}{4 \sqrt{k}}, X_{1}, \ldots, X_{k}\right) \cap K \subset \beta \sqrt{\frac{n}{k}} D
$$

with probability larger than $1-2 e^{-\alpha k}$.

Proof. Let $\varrho>0$ and $x_{0} \in \mathbb{R}^{n}$ be such that $\left|x_{0}\right|=\varrho$. We first apply Theorem 2 to get

$$
\mathrm{Cyl}_{k}\left(\frac{\varrho}{4 \sqrt{n}}, X_{1}, \ldots, X_{k}\right) \cap B\left(x_{0}, c^{\prime}(a) \varrho \sqrt{\frac{k}{n}}\right)=\emptyset
$$

with probability larger than $1-2 e^{-k c(a)}$. Now we use this reasoning for the union of $e^{\alpha k}$ balls centered on a net, to see that if the covering number satisfies

$$
N\left(K \cap \varrho S^{n-1}, c^{\prime}(a) \varrho \sqrt{\frac{k}{n}} D\right) \leq e^{\alpha k},
$$


then with probability larger than $1-2 e^{\alpha k} e^{-k c(a)}$,

$$
\operatorname{Cyl}_{k}\left(\frac{\varrho}{4 \sqrt{n}}, X_{1}, \ldots, X_{k}\right) \cap\left(K \cap \varrho S^{n-1}\right)=\emptyset .
$$

Now note that if a star body $C$ containing 0 satisfies $C \cap \varrho S^{n-1}=\emptyset$, then $C \subset \varrho D$. Since $K$ is star shaped and contains 0 , the previous remark applies to $\operatorname{Cyl}_{k}\left(\varrho / 4 \sqrt{n}, X_{1}, \ldots, X_{k}\right) \cap K$. Hence, we have proved that

$$
\mathrm{Cyl}_{k}\left(\frac{\varrho}{4 \sqrt{n}}, X_{1}, \ldots, X_{k}\right) \cap K \subset \varrho D .
$$

Finally, set $\varrho=\sqrt{n / k} / c^{\prime}(a), \alpha=c(a) / 2$ and $\beta=1 / c^{\prime}(a)$.

REMARK. It is worthwhile to observe that because we deal with the non-centrally symmetric case, the probability $p$ defined by (7) is smaller than $1 / 2$. Therefore, in the proof of the theorem,

$$
\mathbb{P}\left[Z_{1}+\ldots+Z_{k} \leq m\right] \geq(1-p)^{m} \geq 1 / 2^{m} \geq 1 / 2^{k} .
$$

Considering symmetric cut off by hyperplanes yields a completely different and much stronger quantitative statement than Theorem 2. Indeed, the proof now involves an estimate of $\mathbb{P}\left\{\left|\left(x_{0}, X\right)\right|>v\left|x_{0}\right| / \sqrt{n}\right\}$ which may be chosen near 1 . In that case, the parameter $\alpha$ is arbitrary. Below we formulate the corresponding statement concerning symmetric cut off, but we omit the proof as it runs along similar lines to the one above.

Corollary 4. Let $1 \leq k \leq n$. Let $a>0$ and let $X_{1}, \ldots, X_{k} \in M_{n}(a)$ be i.i.d. random vectors. For every $\alpha>0$, there exists $\beta=\beta(a, \alpha)>0$ such that if $K$ is any compact star subset of $\mathbb{R}^{n}$ with 0 in its interior and such that $N(K, D) \leq e^{\alpha k}$, then

$$
\bigcap_{1 \leq i \leq k}\left\{x \in \mathbb{R}^{n} ;\left|\left(x, X_{i}\right)\right| \leq \frac{\beta}{4 \sqrt{k}}\right\} \cap K \subset \beta \sqrt{\frac{n}{k}} D
$$

with probability larger than $1-e^{-\alpha k}$.

Theorem 3 gives rise to a natural dual statement:

Theorem 5. Let $1 \leq k \leq n$. Let $a>0$ and let $X_{1}, \ldots, X_{k} \in M_{n}(a)$ be i.i.d. random vectors. There exist $\alpha=\alpha(a)>0$ and $\beta=\beta(a)>0$ such that if $K$ is any compact star subset of $\mathbb{R}^{n}$ with 0 in its interior and such that $N\left(K^{\circ}, D\right) \leq e^{\alpha k}$, then

$$
\frac{1}{\beta} \sqrt{\frac{k}{n}} D \subset \operatorname{conv}\left(\frac{4 \sqrt{k}}{\beta}\left\{X_{1}, \ldots, X_{k}\right\} \cup K\right)
$$

with probability larger than $1-2 e^{-\alpha k}$. 
5. Applications. From the above two theorems, we can see the following interesting fact. Fix $\varepsilon>0$. Then every $n$-dimensional convex body can be transformed, after a small number $k=\varepsilon n$ of operations (small with respect to the dimension), first by intersection with half-spaces of type $(\cdot, u) \leq c / \sqrt{k}$ and then by taking the convex hull with a small number $k$ of points with Euclidean norm $c \sqrt{k}$, into a body which is close to the convex hull of a Euclidean ball and a $k$-dimensional simplex. Indeed, for $k$ proportional to $n$, there exists a linear transformation of the body $K$, the so-called $M$-position, for which Theorems 3 and 5 may be applied. Dually of course, every convex body can be transformed, by an equally small number of such operations, into a body which is close to the intersection of a Euclidean ball and a small number of slabs or half-spaces.

Theorem 3 and Corollary 4 lead to different patterns. In the first case, non-symmetric, we get the convex hull of a Euclidean ball and $k$ almost orthogonal vectors of norm of order $\sqrt{n}$; in the second case, the convex hull of a Euclidean ball and the $\sqrt{n}$ homothety of a $k$-dimensional $\ell_{1}^{k}$ ball in $\mathbb{R}^{n}$.

It is worthwhile to notice that in order to apply Theorem 3 and obtain a cylinder as intersection of half-spaces, we need accurate information about entropy; that is, the asymptotic behaviour of $N(K, t D)$ as $t$ grows or, at least, the size of $t$ for which $N(K, t D)$ will come below the level $e^{\alpha k}$, which is critical in Theorem 3. Corollary 4 gives a weaker conclusion, since it constructs intersection of symmetric slabs, but we need much less information about the entropy function.

The following statement is an application of Theorem 3. In the case when the $X_{i}$ are uniformly distributed on the unit Euclidean sphere, it should be seen as a global form of well known low- $M^{*}$ estimates (see [M4], [P-T], [G]).

Corollary 6. Let $1 \leq k \leq n$. Let $a>0$ and let $X_{1}, \ldots, X_{k} \in M_{n}(a)$ be i.i.d. random vectors. There exist $\alpha=\alpha(a)>0$ and $\beta=\beta(a)>0$ such that for any compact star subset $K$ of $\mathbb{R}^{n}$ with 0 in its interior and half mean width $M^{*}=M^{*}(K)$, we have

$$
\operatorname{Cyl}_{k}\left(\frac{\sqrt{n / k} \beta M^{*}}{4 \sqrt{k}}, X_{1}, \ldots, X_{k}\right) \cap K \subset \frac{n}{k} \beta M^{*} D
$$

with probability larger than $1-2 e^{-\alpha k}$.

Proof. Recall the Sudakov inequality [Su]: there exists a numerical constant $c>0$ such that for every $t>0$,

$$
N(K, t D) \leq \exp \left(c n\left(M^{*} / t\right)^{2}\right) .
$$

Specifying $t=M^{*} \sqrt{c n / \alpha k}$, we infer that $N\left(t^{-1} K, D\right)=N(K, t D) \leq e^{\alpha k}$. Thus $K_{t}=t^{-1} K$ satisfies the assumption of Theorem 3 and the result follows from Theorem 3 by a standard homogeneity argument. 
Remarks. (i) The classical form of the low- $M^{*}$ estimate follows by considering $E=\bigcap_{1 \leq i \leq k}\left\{x ;\left(x, X_{i}\right)=0\right\}$. When the law of the $X_{i}$ has no atom, $E$ is almost surely a codimension $k$ subspace contained in the cylinder $\operatorname{Cyl}_{k}\left(\frac{\sqrt{n / k} \beta M^{*}}{4 \sqrt{k}}, X_{1}, \ldots, X_{k}\right)$. Therefore its intersection with $K$ is in $(n / k) \beta M^{*} D$, which is the so-called low- $M^{*}$ estimate.

(ii) The previous result, with a slightly different hypothesis, is in the spirit of Proposition 3.3 of [M-S], where symmetric slabs are involved. Classical estimates in this spirit, involving the parameter $M^{*}$, suggest that the estimate $(n / k) M^{*}$ may not be optimal. At least for convex bodies $K$, we would expect here $\sqrt{n / k} M^{*}$, in accordance with [P-T], which corresponds to the case of the rotation invariant measure on $S^{n-1}$. Actually, in Corollary 6 , our set is embedded in $\sqrt{n / k} M^{*} D$, which would recover the best estimates mentioned above. The standard way to achieve this uses an additional piece of information. It is known that if we consider a random projection of rank $k$ of a convex body, then its diameter, with high probability, shrinks by a factor of $\sqrt{k / n}$ up to the time that it achieves $M^{*}$.

(iii) However, an important application we have in mind is the case of uniform counting measure on the vertices of the cube $\{ \pm 1\}^{n}$. Such form of the low- $M^{*}$ estimate was not known to us before. Note that again it may be improved to $\sqrt{n / k} M^{*}$ using the same idea as in (ii). However, this time it uses a recent result of $[\mathrm{A}]$ stating that under some Rademacher projection type operation of rank $k$, with high probability the diameter of a convex body shrinks by a factor $\sqrt{k / n}$ till achieving $M^{*}$.

The next result combines Theorem 3 and its dual form. It shows that $k$ random cut offs by half-spaces at distance of order $1 / \sqrt{k}$ from the origin and the dual operation, convex hull with $k$ random points of Euclidean norm of order $\sqrt{k}$, reduce isomorphically any symmetric convex body to a generic pattern: the convex hull of the unit Euclidean ball and $k$ almost orthogonal vectors of Euclidean norm of order $\sqrt{k}$.

Theorem 7. Let $0<\varepsilon<1$, let $n$ be an integer and set $k=[\varepsilon n]$. Let $a>0$ and let $X_{1}, \ldots, X_{k}, Y_{1}, \ldots, Y_{k} \in M_{n}(a)$ be i.i.d. random vectors. Let $X=\left( \pm X_{1}, \ldots, \pm X_{k}\right)$. There exist $c=c(a, \varepsilon), c^{\prime}=c^{\prime}(a, \varepsilon), \lambda=\lambda(a, \varepsilon)$ and $\lambda^{\prime}=\lambda^{\prime}(a, \varepsilon)>0$ such that for any convex compact subset $K$ of $\mathbb{R}^{n}$, with 0 in its interior, and such that $D$ is an M-ellipsoid for $K$, we have

$$
\lambda E \subset \operatorname{conv}\left(\operatorname{Cyl}_{k}(1 / \sqrt{c k}, X) \cap K, \pm \sqrt{c k} Y_{1}, \ldots, \pm \sqrt{c k} Y_{k}\right) \subset \lambda^{\prime} E
$$

with probability larger than $1-e^{-c^{\prime} n}$, where

$$
E=\operatorname{conv}\left(D, \pm \sqrt{c k} Y_{1}, \ldots, \pm \sqrt{c k} Y_{k}\right) .
$$

Proof. We will need two technical facts concerning a uniform bound for the norm of the vectors $\left|X_{i}\right|$ and the covering number of their convex hull. 
First let $X_{1} \in M_{n}(a)$. As in the proof of (5) in Lemma 1, we have

$$
\left|X_{1}\right| \leq 2 \max \left\{\left(X_{1}, x\right) ; x \in \Lambda(n)\right\}
$$

where $\Lambda(n)$ is a $1 / 2$-net of the unit ball of $\ell_{2}^{n}$ in its own metric and with cardinality less than $5^{n}$. From (3), it follows that for every $v>0$,

$$
\mathbb{P}\left(\left|X_{1}\right|>v\right) \leq 2 \cdot 5^{n} e^{-n(v / 2)^{2} / 2 a^{2}} .
$$

We conclude that there exists $v=v(a)>0$ such that if $X_{1}, \ldots, X_{k} \in M_{n}(a)$ are random vectors, we have

$$
\mathbb{P}\left(\forall i \leq k,\left|X_{i}\right| \leq v\right) \geq 1-e^{-n} .
$$

Now let $A$ be the convex hull of $2 k$ vectors $\pm u_{1}, \pm u_{2}, \ldots, \pm u_{k}$ of Euclidean norm less than $v \sqrt{k}$. It is known ([C-P]) that for any integer $m \geq 1$,

$$
N\left(A, d v \sqrt{\frac{k}{m} \log \left(\frac{k}{m}+1\right)} D\right) \leq e^{m}
$$

for some number $d=d(v)$. Therefore, for any $\alpha>0$, there exists $d(a, \alpha)>0$ such that

$$
N\left(\frac{A}{d(a, \alpha)}, D\right) \leq e^{\alpha k / 2} .
$$

We now proceed to the proof of the theorem. Since $D$ is an $M$-ellipsoid for $K$, it follows that there exists $\alpha=\alpha(\varepsilon)>0$ such that

$$
N(K, D) \leq e^{\alpha k / 2}, \quad N\left(K^{\circ}, D\right) \leq e^{\alpha k / 2} .
$$

We first apply Corollary 4 to $K$ that satisfies $N(K, D) \leq e^{\alpha k}$. We deduce that there exists $\beta=\beta(a, \alpha)>0$ such that

$$
\mathrm{Cyl}_{k}\left(\frac{\beta}{4 \sqrt{k}}, \pm X_{1}, \ldots, \pm X_{k}\right) \cap K \subset \beta \sqrt{\frac{n}{k}} D
$$

with probability larger than $1-e^{-\alpha k}$. This can be written as

$$
B=\operatorname{Cyl}_{k}\left(\frac{1}{\sqrt{k}}, \pm X_{1}, \ldots, \pm X_{k}\right) \cap(4 K / \beta) \subset 4 \sqrt{\frac{n}{k}} D .
$$

We will now restrict $X_{1}, \ldots, X_{k}$ to the subset where $\left|X_{i}\right| \leq v$ for all $i \leq k$, which is of probability larger than $1-e^{-n}$. This restriction may only affect the constant $c^{\prime}$, when computing the probability for which the conclusion of the theorem is valid.

Now $B^{\circ}$ is the convex hull of $\pm \sqrt{k} X_{1}, \ldots, \pm \sqrt{k} X_{k}$ and of $(4 K / \beta)^{\circ}$. The first set is of the type studied above and may be denoted by $A$ so that

$$
B^{\circ} \subset A+(\beta / 4) K^{\circ} \text {. }
$$

Since

$$
N\left(\frac{A}{d(a, \alpha)}, D\right) \leq e^{\alpha k / 2}, \quad N\left(K^{\circ}, D\right) \leq e^{\alpha k / 2},
$$


it follows that

$$
N\left(\frac{A}{2 d(a, \alpha)}+\frac{K^{\circ}}{2}, D\right) \leq e^{\alpha k}
$$

Therefore there exists $c^{\prime \prime}=c^{\prime \prime}(\alpha, \beta)$ such that $N\left(\left(c^{\prime \prime} B\right)^{\circ}, D\right) \leq e^{\alpha k}$. Recall that $(t B)^{\circ}=t^{-1} B^{\circ}$. Applying Corollary 4 to $\left(c^{\prime \prime} B\right)^{\circ}$ and using duality, we find that

$$
\left.\frac{1}{4} \sqrt{\frac{k}{n}} D \subset \operatorname{conv}\left(\sqrt{k}\left\{ \pm Y_{1}, \ldots \pm Y_{k}\right)\right\} \cup\left(\frac{\beta}{4} c^{\prime \prime} B\right)\right)
$$

with probability larger than $1-e^{-\alpha k}$. The final result is obtained after normalization and using homogeneity.

For centrally symmetric bodies, a result with non-symmetric slabs can be obtained. We will say that $D$ is a regular $M$-ellipsoid (of order 1 ) for a convex body $K$ if $|D|=|K|$ and there exists a constant $c>0$ such that for any $t>0$,

$$
N(K, t D) \leq e^{c n / t}, \quad N(D, t K) \leq e^{c n / t} .
$$

It is proved in [M1] and [Pi] that any centrally symmetric convex body has an affine image $K^{\prime}$ such that $D$ is a regular $M$-ellipsoid for $K^{\prime}$. Pisier shows that the same result is true for any order $0<\alpha<2$, meaning that the entropy is not greater than $e^{c n / t^{\alpha}}$ for any $t>0$ [Pi, Corollary 7.15].

Theorem 8. Let $0<\varepsilon<1$, let $n$ be an integer and set $k=[\varepsilon n]$. Let $a>0$ and let $X_{1}, \ldots, X_{k}, Y_{1}, \ldots, Y_{k} \in M_{n}(a)$ be i.i.d. random vectors. Let $X=\left(X_{1}, \ldots, X_{k}\right)$. There exist $c=c(a, \varepsilon), c^{\prime}=c^{\prime}(a, \varepsilon), \lambda=\lambda(a, \varepsilon)$ and $\lambda^{\prime}=\lambda^{\prime}(a, \varepsilon)>0$ such that for any centrally symmetric convex compact subset $K$ of $\mathbb{R}^{n}$, with 0 in its interior, and such that $D$ is a regular $M$ ellipsoid for $K$, we have

$$
\lambda E \subset \operatorname{conv}\left(\operatorname{Cyl}_{k}(1 / \sqrt{c k}, X) \cap K, \sqrt{c k} Y_{1}, \ldots, \sqrt{c k} Y_{k}\right) \subset \lambda^{\prime} E
$$

with probability larger than $1-4 e^{-c^{\prime} n}$, where

$$
E=\operatorname{conv}\left(D, \sqrt{c k} Y_{1}, \ldots, \sqrt{c k} Y_{k}\right) .
$$

Proof. Let $\alpha$ and $\beta$ be as in Theorems 3 and 5. Since $D$ is an $M$-ellipsoid for $K$, there exists $c(\alpha, \varepsilon)>0$ such that

$$
N(K, c(\alpha, \varepsilon) D) \leq e^{\alpha k / 2}, \quad N\left(K^{\circ}, c(\alpha, \varepsilon) D\right) \leq e^{\alpha k / 2} .
$$

We first apply Theorem 3 to $K / c(\alpha, \varepsilon)$ that satisfies $N(K / c(\alpha, \varepsilon), D) \leq e^{\alpha k}$. We get

$$
\mathrm{Cyl}_{k}\left(\frac{\beta}{4 \sqrt{k}}, X_{1}, \ldots, X_{k}\right) \cap(K / c(\alpha, \varepsilon)) \subset \beta \sqrt{\frac{n}{k}} D
$$

with probability larger than $1-2 e^{-\alpha k}$ and with the constant $\beta$ specified by 
these theorems. This can be written as

$$
B=\operatorname{Cyl}_{k}\left(\frac{1}{\sqrt{k}}, X_{1}, \ldots, X_{k}\right) \cap(4 K / \beta c(\alpha, \varepsilon)) \subset 4 \sqrt{\frac{n}{k}} D .
$$

Now $B^{\circ}$ is the convex hull of $\sqrt{k} X_{1}, \ldots, \sqrt{k} X_{k}$ and of $(4 K / \beta c(\alpha, \varepsilon))^{\circ}$. As in the preceding proof and using the same notation, we will now restrict $X_{1}, \ldots, X_{k}$ to the subset where $\left|X_{i}\right| \leq v$ for all $i \leq k$. Recall that this subset has probability larger than $1-e^{-n}$. The first set is the convex hull of less than $2 k$ points of norms less than $v \sqrt{k}$. Denote it by $A$, so that

$$
B^{\circ} \subset A+(\beta c(\alpha, \varepsilon) / 4) K^{\circ} \text {. }
$$

As in the proof above,

$$
N\left(\frac{A}{d(a, \alpha)}, D\right) \leq e^{\alpha k / 2}, \quad N\left(K^{\circ}, c(\alpha, \varepsilon) D\right) \leq e^{\alpha k / 2} .
$$

Therefore

$$
N\left(\frac{A}{2 d(a, \alpha)}+\frac{K^{\circ}}{2 c(\alpha, \varepsilon)}, D\right) \leq e^{\alpha k}
$$

and thus there exists $c^{\prime \prime}=c^{\prime \prime}(a, \alpha, \varepsilon)$ such that $N\left(\left(c^{\prime \prime} B\right)^{\circ}, D\right) \leq e^{\alpha k}$. Applying Theorem 5 to $c^{\prime \prime} B$ shows that

$$
\frac{1}{4} \sqrt{\frac{k}{n}} D \subset \operatorname{conv}\left(\sqrt{k}\left\{Y_{1}, \ldots, Y_{k}\right\} \cup(\beta / 4) c^{\prime \prime} B\right)
$$

with probability larger than $1-2 e^{-\alpha k}$. The final result is obtained after normalization and using homogeneity.

REMARK. Let $k=[\varepsilon n]$ as above and let $Y_{1}, \ldots, Y_{k}$ be i.i.d. uniformly distributed on the sphere or Gaussian vectors with covariance matrix $n^{-1} I$. Let $T$ be the operator from $\ell_{2}^{k}$ onto the linear subspace of $\ell_{2}^{n}$ generated by $Y_{1}, \ldots, Y_{k}$, mapping the canonical basis onto $Y_{1}, \ldots, Y_{k}$. It is known (see [D-S]) that $\|T\| \cdot\left\|T^{-1}\right\|$ is bounded for $\varepsilon<1 / 2$. As a consequence, regularization using a "cubical" cylinder as in Corollary 4 leads to a canonical pattern $E=\operatorname{conv}\left(D \cup \sqrt{n} B_{1}^{k}\right)$, where $B_{1}^{k}$ denotes the unit ball of $\ell_{1}^{k}$.

The next application concerns another important geometric parameter, the so-called volume ratio.

TheOREM 9. Let $0<\varepsilon<1$, let $n$ be an integer and set $k=[\varepsilon n]$. Let $a>0$ and let $X_{1}, \ldots, X_{k} \in M_{n}(a)$ be i.i.d. random vectors. Let $r>0$. There exist $c=c(a, \varepsilon, r)$ and $c^{\prime}=c^{\prime}(a, \varepsilon, r)>0$ such that for any convex body $K$ in $\mathbb{R}^{n}$ satisfying $D \subset K$ and $|K| /|D| \leq r^{n}$, we have

$$
\bigcap_{1 \leq i \leq k}\left\{x \in \mathbb{R}^{n} ;\left|\left(x, X_{i}\right)\right| \leq \frac{c}{4 \sqrt{k}}\right\} \cap K \subset c D
$$

with probability larger than $1-e^{-c^{\prime} n}$. 
Proof. Let $K^{\prime}=K-K$. From [R-S] we have $\left|K^{\prime}\right| \leq\left(\begin{array}{c}2 n \\ n\end{array}\right)|K| \leq 4^{n}|K|$. Since $D \subset K^{\prime}$, for any $t>0$ one has (see [M-S])

$$
N\left(K^{\prime}, t D\right) \leq\left(1+\frac{2}{t}\right)^{n}(4 r)^{n}
$$

Since $K \subset K^{\prime}$, the result follows from Corollary 4 .

REMARK. With the previous notation, considering the linear space $E=$ $\bigcap_{1 \leq i \leq k}\left\{x \in \mathbb{R}^{n} ;\left(x, X_{i}\right)=0\right\}$ with dimension larger than $(1-\varepsilon) n$, we recover a well known result from [S-T] on large nearly Euclidean sections of finite volume-ratio spaces. However, Theorem 9 gives a global form. Note that choosing the $X_{i}$ to be, for example, vectors with \pm 1 coordinates gives a special kind of Euclidean section.

\section{References}

[A] S. Artstein, The diameter of sign-projections of convex bodies, GAFA Seminar, Lecture Notes in Math., Springer, to appear.

[B-N1] S. Bobkov and F. L. Nazarov, On convex bodies and log-concave probability measures with unconditional basis, in: Geometric Aspects of Functional Analysis (2000-2002), V. Milman and G. Schechtman (eds.), Lecture Notes in Math. 1807, Springer, 2003, 53-69.

[B-N2] - - - Large deviations of typical linear functionals on a convex body with unconditional basis, preprint.

[B-K] F. Barthe and A. Koldobsky, Extremal slabs in the cube and the Laplace transform, Adv. Math., to appear.

[C-P] B. Carl and A. Pajor, Gelfand numbers of operators with values in a Hilbert space, Invent. Math. 94 (1988), 479-504.

[D-S] K. R. Davidson and S. Szarek, Local operator theory, random matrices and Banach spaces, in: Handbook of the Geometry of Banach Spaces, Vol. I, W. B. Johnson and J. Lindenstrauss (eds.), Elsevier, 2001, 317-366.

[G] Y. Gordon, On Milman's inequality and random subspaces which escape through a mesh in $\mathbb{R}$, in: Geometric Aspects of Functional Analysis (1986/87), Lecture Notes in Math. 1317, Springer, Berlin, 1988, 84-106.

[M1] V. Milman, An inverse form of the Brunn-Minkowski inequality with applications to local theory of normed spaces, C. R. Acad Sci. Paris Sér. I 302 (1986), 25-28.

[M2] - Isomorphic symmetrizations and geometric inequalities, in: Geometric Aspects of Functional Analysis (1986/87), Lecture Notes in Math. 1317, Springer, 1988, 107-131.

[M3] - Surprising geometric phenomena in high-dimensional convexity theory, in: European Congress of Mathematics, Vol. II (Budapest, 1996), Progr. Math. 169, Birkhäuser, Basel, 1998, 73-91.

[M4] - Almost Euclidean quotient spaces of subspaces of a finite dimensional normed space, Proc. Amer. Math. Soc. 94 (1985), 445-449.

[M-P1] V. Milman and A. Pajor, Entropy and asymptotic geometry of non-symmetric convex bodies Adv. Math. 152 (2000), 314-335. 
[M-P2] V. Milman and A. Pajor, Isotropic position and inertia ellipsoids and zonoids of the unit ball of a normed n-dimensional space, in: Lecture Notes in Math. 1376, Springer, Berlin, 1989, 64-104.

[M-P3] -, -, Entropy method in asymptotic convex geometry, C. R. Acad. Sci. Paris 329 (1999), 303-308.

[M-S] V. Milman and S. Szarek, A geometric lemma and duality of entropy numbers, in: Geometric Aspects of Functional Analysis, Israel Seminar 1996-2000, V. D. Milman and G. Schechtman (eds.), Lecture Notes in Math. 1745, Springer, Berlin, 2000, 191-222.

[P-T] A. Pajor and N. Tomczak-Jaegermann, Subspaces of small codimension of finitedimensional Banach spaces, Proc. Amer. Math. Soc. 97 (1986), 637-642.

[Pi] G. Pisier, The Volume of Convex Bodies and Banach Space Geometry, Cambridge Univ. Press, 1989.

[R-S] C. A. Rogers and G. C. Shephard, The difference body of a convex body, Arch. Math. (Basel) 8 (1957), 220-233.

[Su] V. N. Sudakov, Gaussian processes and measures of solid angles in Hilbert spaces, Soviet Math. Dokl. 12 (1971), 412-415.

[S-T] S. Szarek and N. Tomczak-Jaegermann, On nearly Euclidean decompositions of some classes of Banach spaces, Compositio Math. 40 (1980), 367-385.

Department of Mathematics

Tel Aviv University

Ramat Aviv, Israel

E-mail: vitali@math.tau.ac.il
Équipe d'Analyse et Mathématiques Appliquées Université de Marne-la-Vallée 5 boulevard Descartes, Champs sur Marne 77454 Marne-la-Vallée Cedex 2, France E-mail: pajor@math.univ-mlv.fr

Received November 12, 2002

Revised version March 5, 2003 